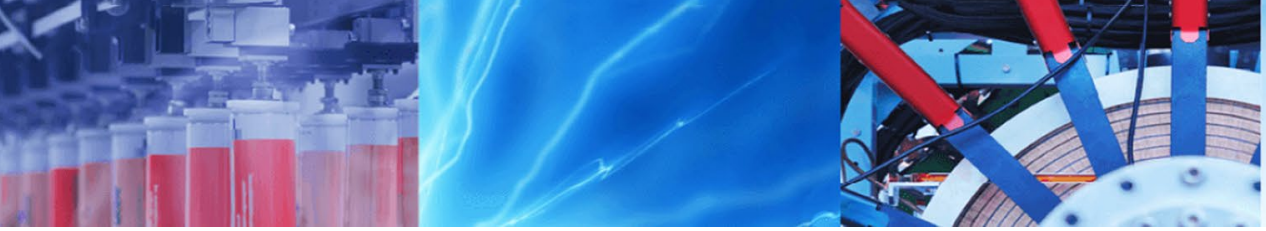

Short communication

\title{
Tensile behavior of tailor rolled blanks: mathematical modeling and design optimization
}

\author{
E. Shafiei ${ }^{1}$ (1) A. Soltani Tehrani ${ }^{2} \cdot$ F. Ostovan ${ }^{1}$
}

(c) Springer Nature Switzerland AG 2018

\begin{abstract}
In this study, a mathematical model considering geometrical characteristics of thickness transition zone (TTZ) has been developed to predict tensile behavior of tailor rolled blanks (TRBs). To prove the accuracy of the presented model, uniaxial tension tests were carried out using tensile specimens fabricated from TRBs with different TTZ geometries (thickness ratio varies between 0.47 and 0.89 and length of TTZ changes from 58 to $110 \mathrm{~mm}$ ). The error analysis showed that the predicted results correlate well with the ones obtained from uniaxial tension tests. In addition, utilizing the presented model, a new mathematical relation was proposed to obtain the optimized design for TTZ at each thickness ratio. Accordingly, for thickness ratios below 0.5 , it is anticipated that total percent elongation of TRBs increases up to a maximum point with increasing effective length ratio (ELR), characterized by a critical ELR parameter. However, for thickness ratios above 0.5 , the elongation-ELR curves may display a full ascending trend with no peak point within $0<E L R \leq 0.5$. The reasons behind these variations were fully discussed.
\end{abstract}

Keywords Tailor rolled blanks ·TRB · Tensile behavior $\cdot$ Modeling $\cdot$ Total elongation

\section{Introduction}

Environmental concerns, safety regulations and consumer demands are driving the need for a lighter vehicle that is more fuel efficient, produces lower emissions and provides improved crashworthiness and performance reliability [1]. Accordingly, it has become a big challenge for product development teams to create a design to satisfy all the aforementioned expectations. As a result, great attention has been paid to tailor rolled blanks (TRB) with variable thicknesses to produce lightweight components in automotive industry with popular applications including side frames, doors and pillars [2].

Kopp et al. [3] investigated the formability of TRBs utilizing deep drawing tests. They concluded that the longer thickness transition zone (TTZ) results in less wrinkling. In addition, Zhang et al. [4] developed a differential equation to predict rolling force during variable gauge rolling using slab method analysis. Liu et al. [5] presented a kinematically admissible velocity field to analyze forward slip during different stages of variable gauge rolling. Zhang et al. [6] utilized linear Lagrangian interpolation method to estimate the mechanical properties of TTZ at each point. Shafiei and Dehghani [7] studied the effects of thickness ratio and length of TTZ on the tensile behavior of TRBs. They concluded that total elongation of TRBs decreases with decreasing thickness ratio. Furthermore, they found out that increasing length of TTZ has a detrimental effect on the total elongation of TRBs. In a different study, they examined the effects of strength gradient and geometrical inhomogeneity on the total elongation of TRBs. It was concluded that these factors can restrict total elongation of TRBs during uniaxial tension [8]. Duan et al. [9] studied the design optimization of TRB thin-walled structures utilizing genetic algorithm. Accordingly, they

E. Shafiei, shafiei.ehsan.mse@gmail.com | 'Department of Materials Science and Engineering, Bandar Abbas Branch, Islamic Azad University, Bandar Abbas, Hormozgan, Iran. ${ }^{2}$ Department of Mechanical Engineering, Auburn University, Auburn, AL 36849, USA. 
found out that the crashworthiness and lightweight performance of the optimized TRBs are superior to the uniform thickness components. In another study, Sun et al. [10] investigated crashworthiness of the automotive parts made with TRBs using finite element simulations. Accordingly, a new method was established to obtain optimized TRB parts with maximum energy absorption. In the previous researches carried out by the first author of the present study, the effects of thickness transition zone geometry on the total elongation of TRBs were fully discussed; however, lack of a mathematical equation that can be used to predict the tensile behavior of such blanks is still present $[7,8]$.

Since no researches have been conducted on the development of mathematical models for the prediction of tensile behavior of TRBs, the main aim of this study is to propose a mathematical formulation to predict total percent elongation of TRBs obtained from uniaxial tension. To this end, effects of thickness ratio and effective length ratio (ELR) on the tensile behavior of the studied TRBs were considered to develop a comprehensive model. Furthermore, with regard to the importance of design optimization, a critical condition was introduced to optimize the dimensions of TTZ to achieve maximum total percent elongation at each thickness ratio.

\section{Experimental procedure}

The material used for this study was DP590 steel sheets with the initial thickness of $2 \mathrm{~mm}$. The chemical composition of the investigated material is summarized in Table 1. In addition, mechanical properties of the as-received sheet are shown in Table 2.

Variable gauge rolling (VGR) was carried out using a four-high mill having the working roll of $183 \mathrm{~mm}$ in diameter. The roll gap was automatically controlled by a computer to produce TRBs with different thickness ratios ranging from 0.47 to 0.89 with lengths of TTZ changing from 58 to $110 \mathrm{~mm}$. To this end, the rolling machine was equipped with a set of proper hydraulic cylinders as actuators. Accordingly, the thickness amounts at the outlet were monitored using a thickness measurement sensor for feedback control. In case of deviation from the target values, the hydraulic cylinders were driven to adjust the roll gap.

Since there is no specific international standard for tensile specimens of TRBs with longitudinal TTZ, the considerations of ASTM E8M [11] were adopted to obtain the proper dimensions for tensile specimens with variable thicknesses. Figure 1 depicts the dimensions of the tensile specimens fabricated from TRBs with variable TTZ geometries. All the tensile tests were performed at a constant crosshead speed of $3 \mathrm{~mm} / \mathrm{min}$ using an Instron 8803 tensile machine.
Table 1 Chemical compositions (wt\%) of DP590

\begin{tabular}{llllllll}
\hline Elements & $\mathrm{C}$ & $\mathrm{Si}$ & $\mathrm{Mn}$ & $\mathrm{Nb}$ & $\mathrm{Al}$ & $\mathrm{Cr}$ & $\mathrm{Fe}$ \\
\hline wt\% & 0.149 & 0.467 & 1.513 & 0.016 & 0.033 & 0.23 & Bal. \\
\hline
\end{tabular}

Table 2 Mechanical properties of the studied material

\begin{tabular}{|c|c|c|c|c|c|c|c|}
\hline & Yield stress (MPa) & $\begin{array}{l}\text { Ultimate tensile } \\
\text { strength (MPa) }\end{array}$ & $\begin{array}{l}\text { Young's modulus } \\
\text { (GPa) }\end{array}$ & $n$ & $K(\mathrm{MPa})$ & Total elongation (\%) & $\begin{array}{l}\text { Uniform elongation } \\
\text { (\%) }\end{array}$ \\
\hline DP590 & $486 \pm 14$ & $731 \pm 18$ & 207 & $0.125 \pm 0.02$ & $900 \pm 20$ & $27 \pm 0.5$ & $19 \pm 0.5$ \\
\hline
\end{tabular}

Fig. 1 Dimensions of uniaxial tensile specimens made from TRBs

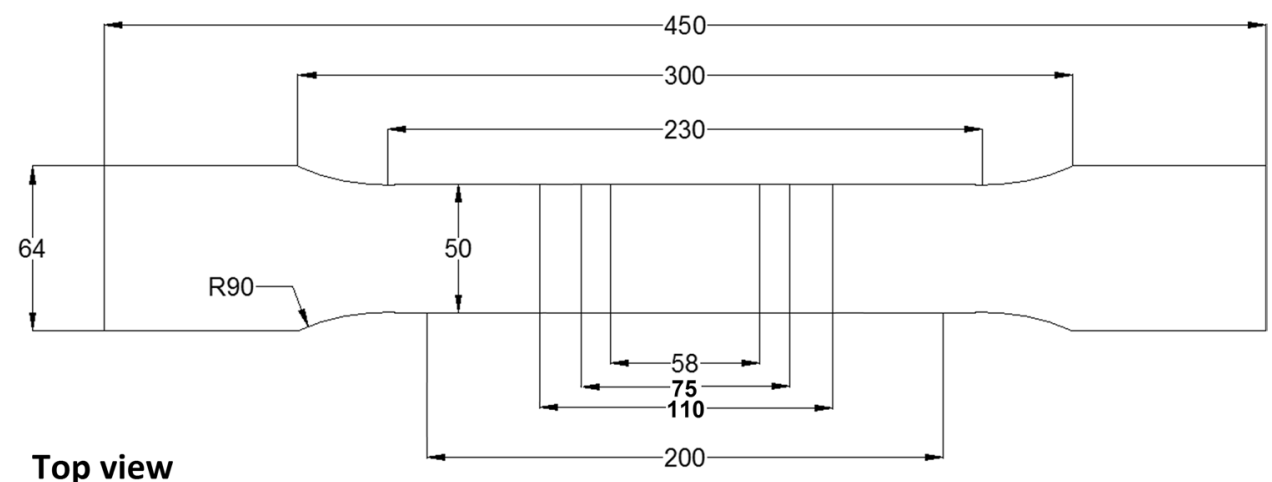




\section{Results and discussion}

Figure 2 shows the changes of the total percent elongation of the studied TRBs with thickness ratio at different lengths of TTZ. As can be seen, total percent elongation of TRBs increases exponentially with increasing thickness ratio. This can be mainly attributed to the changes of the applied stress concentration on the thinner side. On the

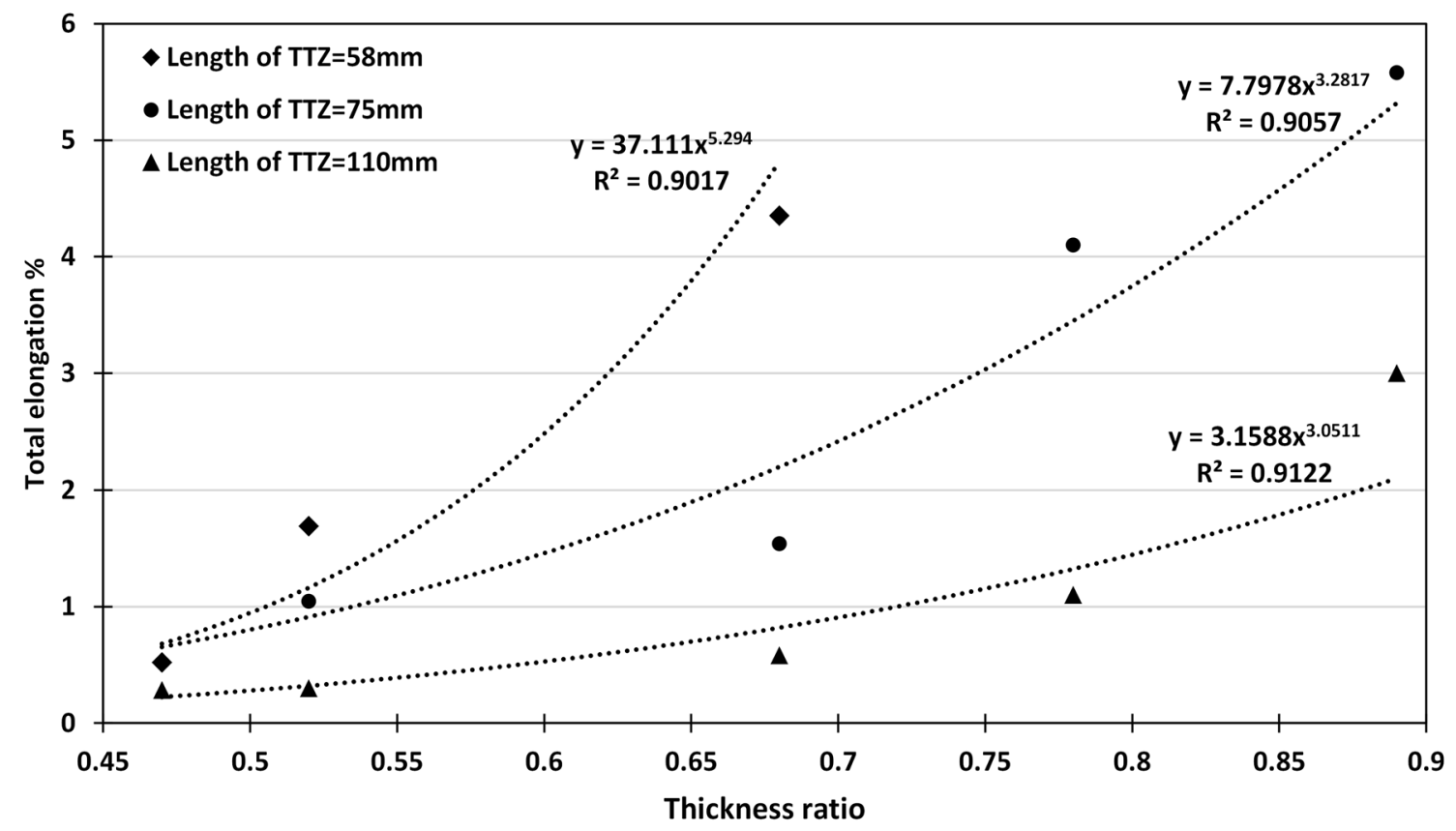

Fig. 2 Effects of thickness ratio and length of TTZ on the total elongation of the studied TRBs

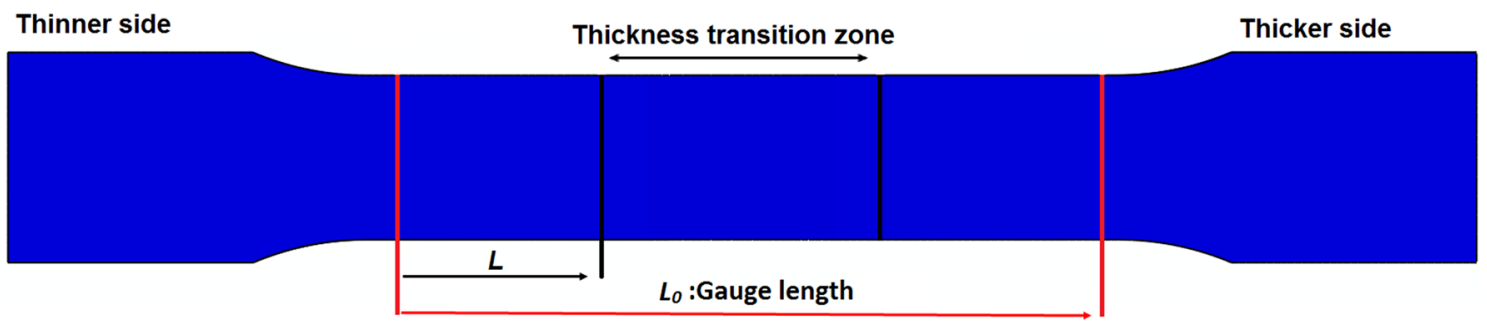

Effective length ratio $=L / L_{0}$

Fig. 3 Definition of effective length ratio (ELR) in tensile specimens fabricated from TRBs with variable thicknesses

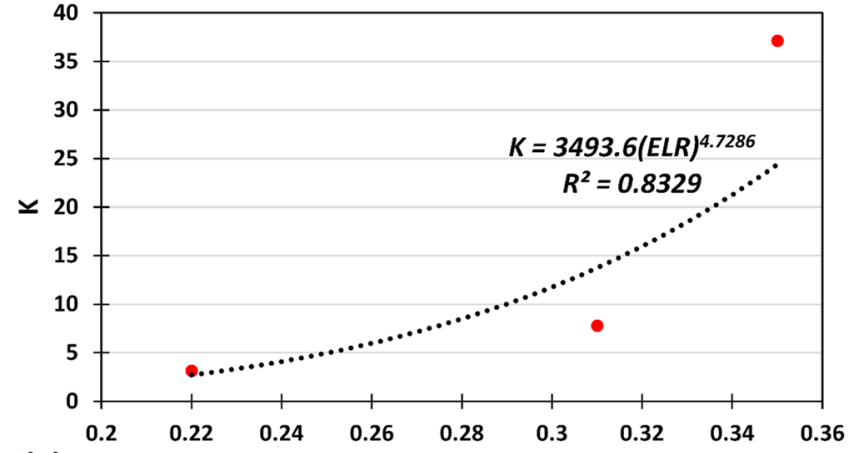

(a)

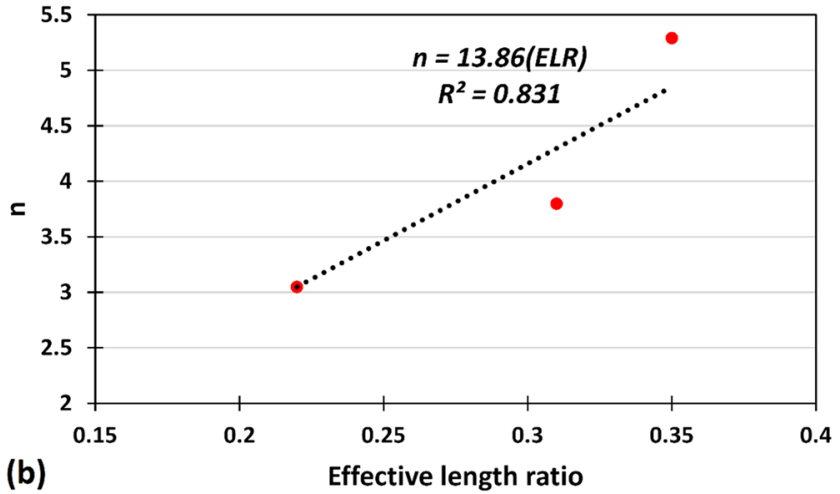

Effective length ratio

Fig. 4 Plots used to obtain material constants 


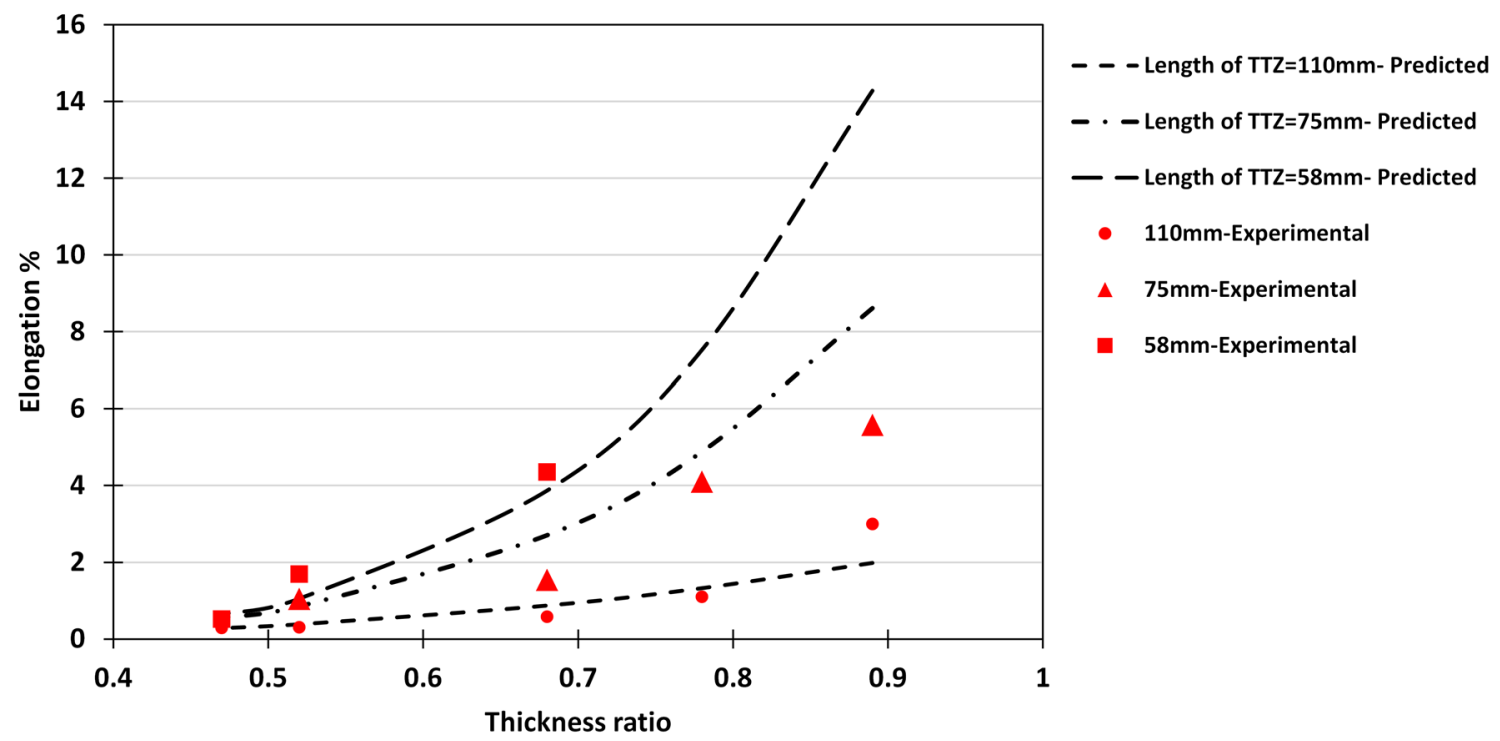

Fig. 5 Comparison between experimental data and predictions of the presented model

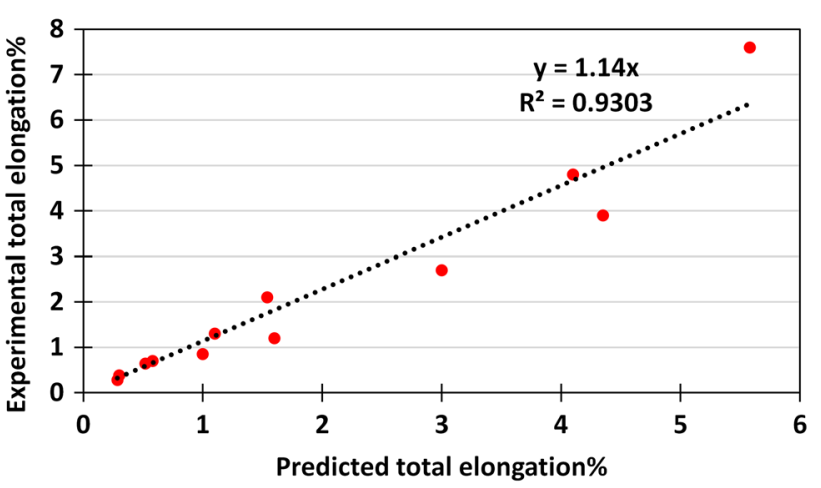

Fig. 6 Experimental data versus predicted ones

one hand, the stress concentration on the thinner side increases by thickness ratio decrement which can restrict the total elongation of TRBs by increasing stress triaxiality factor on the thinner side. On the other hand, formability of the thinner side, as the deformation governing side, decreases with decreasing thickness ratio which can also result in the reduction of total elongation.

Moreover, as can be observed in Fig. 2, total percent elongation decreases with increasing length of TTZ. To justify this behavior, Shafiei and Dehghani [7] have presented a new geometrical parameter named effective length ratio (ELR) recently. They have found that any reduction in ELR can restrict total percent elongation of TRBs due to domination of plane strain condition on the thinner side. In this regard, Fig. 3 shows how to obtain ELR parameter in TRBs schematically. The $L$ and $L_{0}$ parameters in Fig. 3 represent the length of thinner side and gauge length, respectively.
According to the definition of ELR parameter, for symmetrical tensile specimens, the lengths of TTZ of 58,75 and $110 \mathrm{~mm}$ correspond to the ELR parameters of $0.35,0.31$ and 0.22 , respectively. Regarding Fig. 2, variations of total percent elongation of TRBs with thickness ratio can be considered as follows:

$\mathrm{TE} \%=k\left(t_{\mathrm{r}}\right)^{n}$,

where TE\% and $t_{\mathrm{r}}$ are total percent elongation of TRBs at a constant length of TTZ and thickness ratio, respectively. In addition, $k$ and $n$ are constants related to the material, which considerably change with ELR parameter. As can be deduced from the data provided in Fig. 2, the degree to which the total percent elongation is dependent on thickness ratio is highly reliant on the length of TTZ (or on the ELR parameter, as defined in Fig. 3). In other words, at low ELR values (or high length of TTZ), the total percent elongation is restricted by plane strain condition on the thinner side and consequently the slope of total elongation- thickness ratio curve is less sensitive to the variations of thickness ratio. On the contrary, at high ELR values (or low length of TTZ), the stress triaxiality along TTZ which is mainly controlled by thickness ratio can restrict the total elongation and accordingly the slope of total elongation- thickness ratio curves changes more rapidly with thickness ratio. Accordingly, constants $n$ and $k$ were considered as ELR-sensitive parameters and their types of dependency on ELR parameter were determined utilizing experimental data. Hence, considering the effect of ELR parameter on total percent elongation, Eq. (1) can be rewritten in the following form: 

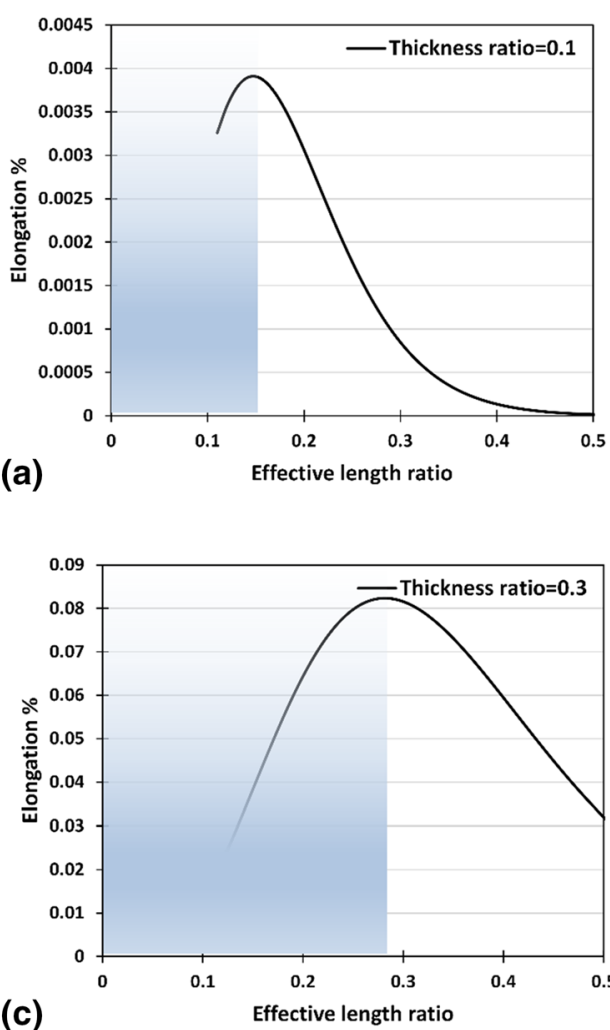

Critical condition: $E L R=0.28$

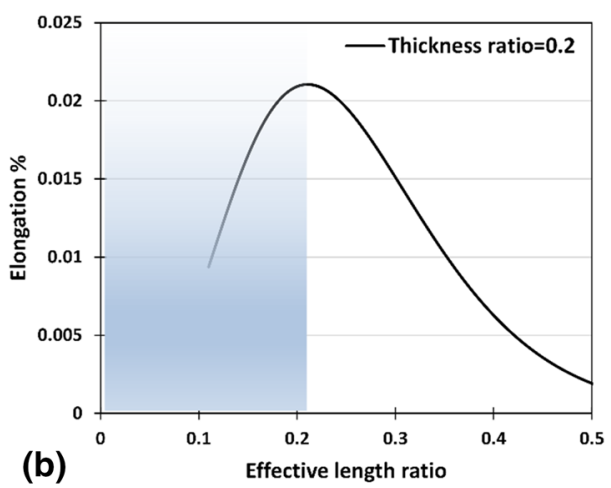

Critical condition: $\mathrm{ELR}=0.21$

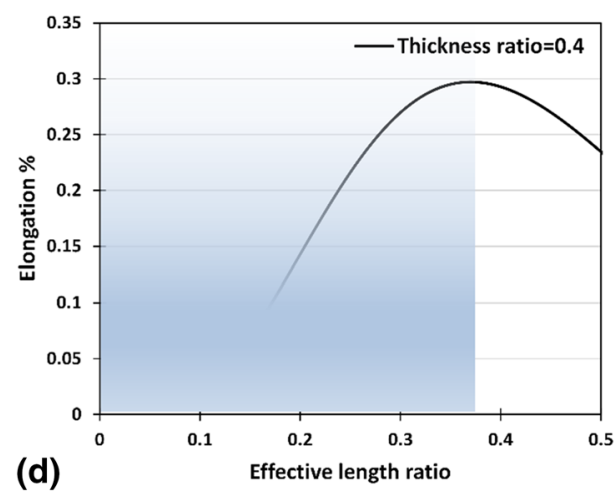

Critical condition: $\mathrm{ELR}=0.37$

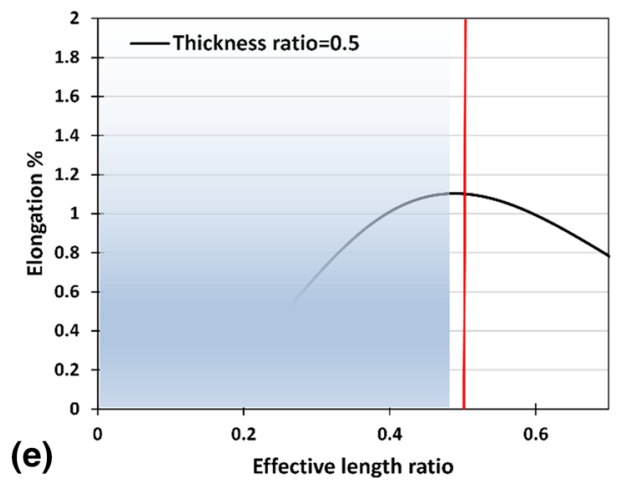

Critical condition: $\mathrm{ELR}=0.48$

Fig. 7 Variations of total percent elongation of TRBs with thickness ratio $\left(t_{\mathrm{r}} \leq 0.5\right)$

$\mathrm{TE} \%=A(\mathrm{ELR})^{m}\left(t_{\mathrm{r}}\right)^{c(\mathrm{ELR})}$,

where $k$ equals to $A(E L R)^{m}$ and constant $n$ equals to $c^{*}$ ELR. Values of constants $A$ and $m$ can be obtained using $k$-ELR plots. In addition, constant $c$ can be calculated by incorporating $n$-ELR plots. It is also noteworthy to mention that ELR maximum value will be equal to 0.5 for the symmetrical tensile specimens fabricated from TRBs.

Using the first derivative of Eq. (2) with respect to ELR, the critical ELR parameter at which the maximum total percent elongation of TRBs is achieved can be expressed in the following form:

$$
\left.E L R\right|_{\text {critical }}=-\frac{m}{c * \ln \left(t_{\mathrm{r}}\right)} .
$$

Accordingly, it can be expected that the total percent elongation of TRBs (at a constant thickness ratio) increases up to a maximum value with increasing ELR parameter to the defined critical values in Eq. (3). However, further increase in the ELR to the values greater than critical ELR may have adverse effects on total percent elongation. In such conditions, deformation will be localized along a short TTZ during uniaxial tension. Consequently, after the 


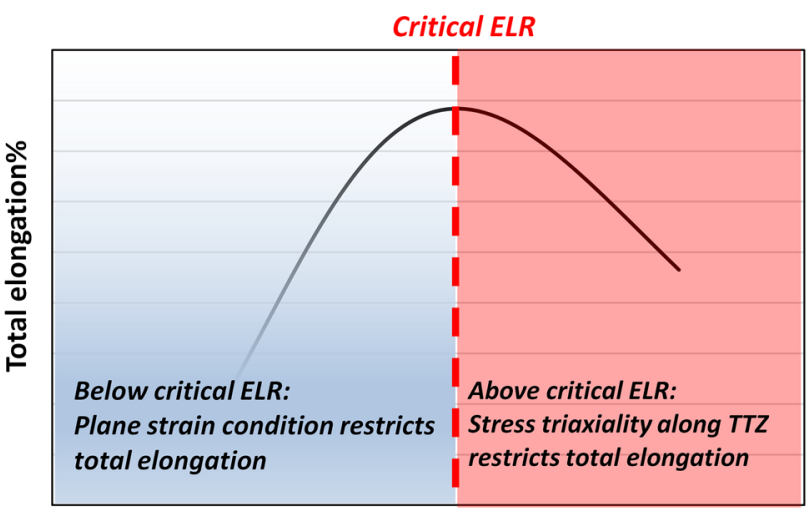

Effective length ratio

Fig. 8 Factors affecting total elongation for thickness ratios below 0.5

peak point, total percent elongation may decrease with increasing ELR.

Figure $4 a$ and $b$ shows the plots used to obtain the constants of Eq. (2). Values of constants $A, m$ and $C$ were calculated as $3493.6,4.72$ and 13.86 for the alloy of this study, respectively. Accordingly, Eqs. (2) and (3) can be rewritten in the following forms:

$\mathrm{TE} \%=3493.6(\mathrm{ELR})^{4.72}\left(t_{\mathrm{r}}\right)^{13.86(\mathrm{ELR})}$

$\left.E L R\right|_{\text {critical }}=-\frac{4.72}{13.86 * \ln \left(t_{\mathrm{r}}\right)}$

Figure 5 shows the predicted total elongations of TRBs in comparison with the experimentally measured ones at different specimens' geometries. As can be seen, the predicted results are in good agreement with the ones
Table 3 Predicted optimized dimensions for TTZ at different thickness ratios

\begin{tabular}{lll}
\hline Thickness ratio & Optimized ELR parameter & $\begin{array}{l}\text { Optimized } \\
\text { length of TTZ } \\
(\mathrm{mm})\end{array}$ \\
\hline 0.1 & 0.15 & 140 \\
0.2 & 0.21 & 116 \\
0.3 & 0.28 & 88 \\
0.4 & 0.37 & 52 \\
0.5 & 0.48 & 8 \\
\hline
\end{tabular}

obtained from uniaxial tension. Accordingly, in order to evaluate the competency of the presented model more accurately, predicted results were plotted against experimental data, as shown in Fig. 6. The low error value as well as the slope of fitted line $(\approx 1)$ specifies a high accordance between the predicted and measured values of total percent elongation of TRBs.

Figure 7a-e shows the predicted changes of total percent elongation with ELR at thickness ratios less than 0.5 using Eqs. (4) and (5). As it can be seen, the ELR critical value increases with increasing thickness ratio. Since the stress-strain fields become more uniform along TTZ during uniaxial tension with increasing thickness ratio up to 0.5 , it would be reasonable to obtain higher values for critical ELR with increasing thickness ratio. Factors affecting tensile behavior of TRBs for thickness ratios less than 0.5 are summarized schematically in Fig. 8.

Figure 9 shows the changes of the total percent elongation as a function of ELR parameter at thickness ratios above 0.5 . As can be seen, at this range, total percent elongation increases continuously with increasing ELR parameter. Accordingly, it can be deduced that because of stress field's more uniformity along TTZ at higher thickness
Fig. 9 Variations of total percent elongation of TRBs with thickness ratio $\left(0.5<t_{\mathrm{r}} \leq 0.9\right)$

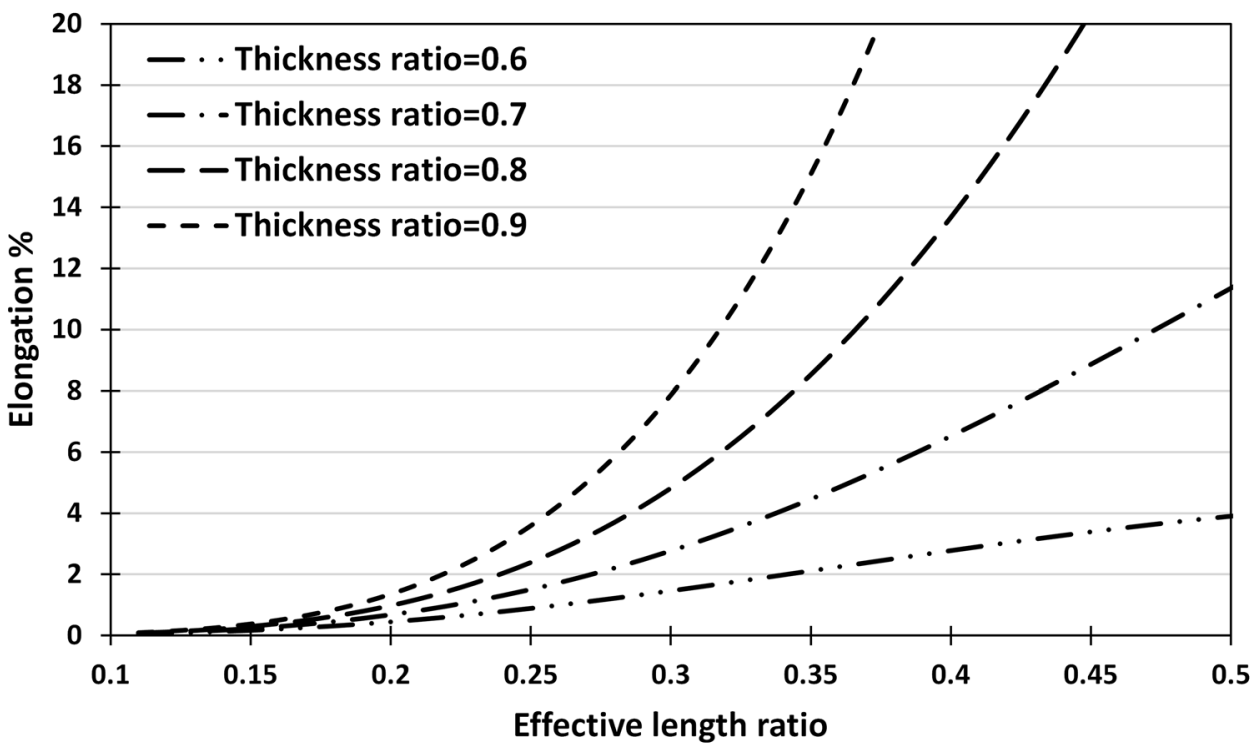


Fig. 10 Predicted total elongation and corresponding experimentally measured ones for thickness ratio of 0.4

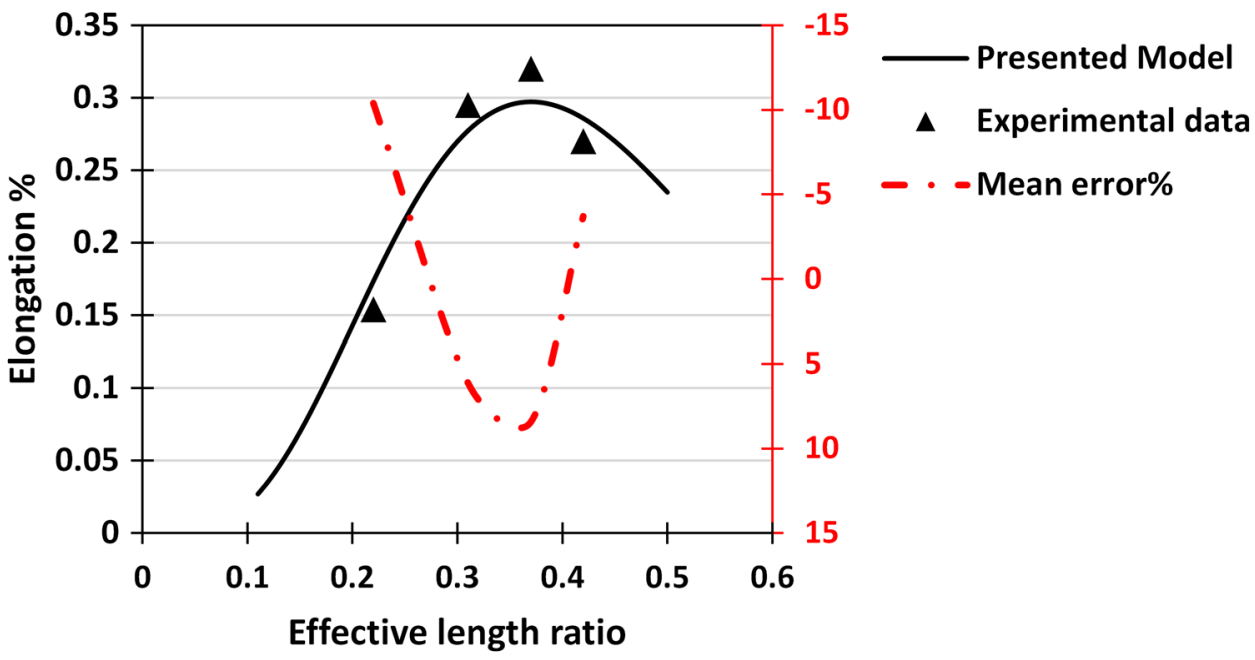

ratios, no critical value (peak point) is expected to be observed for ELRs within $0<E L R \leq 0.5$. These results are theoretically acceptable although extensive experimental works are required to prove these findings at wide thickness ratios, which can be the subject for future researches.

According to the obtained results, optimized dimensions of the TTZ, which yield maximum total percent elongation of TRBs, can be calculated utilizing critical ELR parameter for thickness ratios below 0.5. Optimized dimensions of the TTZ for each thickness ratio are shown in Table 3. Accordingly, it is possible to state that, e.g., at thickness ratio of 0.2 , the total percent elongation increases with increasing the length of TTZ to $116 \mathrm{~mm}$; afterward, the total percent elongation decreases with more increase in the length of TTZ. In addition, in order to prove the validity of the presented method, experimental data obtained from uniaxial tension of TRBs at different ELR parameters and constant thickness ratio of 0.4 were used. As can be seen in Fig. 10, the maximum error of the presented method in the prediction of total elongation of TRBs is about $11 \%$ which lies in an acceptable range. Furthermore, for both experimental data and predicted results, the maximum total percent elongation is achieved at the critical ELR parameter of 0.37 (roughly the same value). Consequently, it can be concluded that these findings have a great potential to be used for fabrication of auto body components from TRBs with optimized tensile behavior. To this end, tensile behavior of TRBs should be modeled first using the presented mathematical relation and then the optimized length of TTZ can be obtained using Eq. (3) to achieve maximum total percent elongation for TRBs with thickness ratios less than 0.5 . This optimization procedure would be beneficial from both lightweight and crashworthiness points of view, especially when a good tensile behavior is expected from TRB components.

\section{Conclusions}

- A mathematical model has been presented to predict total percent elongation of tailor rolled blanks (TRBs) by considering the effects of thickness ratio and effective length parameter (ELR). The results showed that the presented model can give a precise estimation of total percent elongation of TRBs.

- A critical condition has been introduced for variations of total percent elongation with effective length ratio for TRBs with thickness ratios below 0.5. For the ELR values less than the critical value, it is anticipated that total percent elongation increases with increasing ELR. However, for the ELR values above the critical value, total percent elongation may decrease with further increase in ELR.

- For thickness ratios above 0.5 , the elongation-ELR curves may display a full ascending trend with no peak point within $0<\mathrm{ELR} \leq 0.5$.

- According to the optimized dimensions obtained for TTZ, it can be concluded that the optimized length of TTZ decreases with increasing thickness ratio.

\section{Compliance with ethical standards}

Conflict of interest The authors declare that they have no conflict of interest.

\section{References}

1. Chuang CH, Yang RJ, Li G, Mallela K, Pothurajo P (2008) Multidisciplinary design optimization on vehicle tailor rolled blank design. Struct Multidiscp Optim 35:551-560 
2. Shafiei $E$, Dehghai $K$ (2017) Tensile behavior of tailor rolled blanks with longitudinal thickness transition zone: Introducing a new tensile specimen. Vacuum 143:71-86

3. Kopp R, Weidner C, Meyer A (2005) Flexible rolled sheet metal and its use in sheet metal forming. Adv Mater Res 6:81-92

4. Zhang G, Liu X (2012) Research on roll force for variable gauge rolling. Adv Mater Res 418:1232-1236

5. Liu X, Wu Z, Zhi F et al (2012) From TRB and LP plate to variable gauge rolling: technology, theory, simulation and experiment. Mater Sci Forum 706:1448-1453

6. Zhang HW, Liu X, Liu LZ et al (2015) Study on nonuniform deformation of tailor rolled blanks during uniaxial tension. Acta Metall Sin 28:1198-1204

7. Shafiei E, Dehghani K (2018) Effects of thickness ratio and length of thickness transition zone on the tensile behavior of tailor rolled blanks. Trans Indian Inst Met. https://doi.org/10.1007/ s12666-017-1256-x

8. Shafiei E, Dehghani K (2017) Effects of thickness ratio on tensile behavior of tailor rolled blanks. Can Metall Q. https://doi. org/10.1080/00084433.2017.1399235

9. Duan L, Xiao NC, Li G, Chung A, Chen T (2017) Design optimization of tailor-rolled blank thin-walled structures based on -support vector regression technique and genetic algorithm. Eng Optim 49:1148-1165

10. Sun G, Tian J, Lu T, Yan X, Huang X (2018) Crashworthiness optimization of automotive parts with tailor rolled blank. Eng Struct 169:201-215

11. ASTM E8/E8M (2009) Standard test methods for tension testing of metallic materials. https://doi.org/10.1520/e0008_e0008m-09 